\title{
Prevalência da sintomatologia depressiva e capacidade funcional em idosos
}

\author{
Prevalence of depressive symptomatology and functional \\ capacity in elderly
}

\section{Prevalencia de sintomatología depresiva y capacidad funcional en ancianos}

\author{
Joyce Bizerra Mendes ${ }^{1}$, Jessica Bruna Florêncio e Silva ${ }^{2}$, Daniela \\ Maria Silva Maia ${ }^{3}$, Elanny Mirelle da Costa ${ }^{4}$, Ana Clara Sena Bento ${ }^{5}$, \\ Joelma Gomes da Silva ${ }^{6}$
}

\begin{abstract}
1.Bacharel em Fisioterapia pela Faculdade Vale do Jaguaribe, FVJ. Aracati-CE, Brasil. https://orcid.org/0000-0003-3719-3304

2.Acadêmica do curso de Fisioterapia da Faculdade Nova Esperança de Mossoró, FACENE RN. Mossoró-RN, Brasil. https://orcid.org/0000-0002-0203-6248

3.Bacharel em Fisioterapia, Centro Universidade Estácio do Ceará. Especialista em Traumato-Ortopedia com Ênfase em Terapia Manual, Centro Universitário Estácio do Ceará. Docente do Curso de Fisioterapia na Faculdade Vale do Jaguaribe, FVJ. Aracati-CE, Brasil. https://orcid.org/0000-0002-1724-2124

4. Bacharel em Fisioterapia pela Universidade Potiguar, UnP. Especialista em Fisioterapia Neurofuncional pela Faculdade Católica de Mossoró. Docente do Curso de Fisioterapia da Faculdade Nova Esperança de Mossoró, FACENE RN. Mossoró-RN, Brasil. https://orcid.org/0000-0002-2947-7529

5.Acadêmica do curso de Fisioterapia da Faculdade Nova Esperança de Mossoró, FACENE RN. Mossoró-RN, Brasil. https://orcid.org/0000-0001-8227-1944

6.Bacharel em Fisioterapia pela Universidade Estadual da Paraíba, UEPB. Especialista em Fisioterapia Traumato-ortopédica e desportiva pela Universidade Federal do Rio Grande do Norte, UFRN. Mestre em Saúde e Sociedade pela Universidade do Estado do Rio Grande do Norte, UERN. Docente e Coordenadora do Curso de Fisioterapia da Faculdade Nova Esperança de Mossoró, FACENE RN. Mossoró-RN, Brasil. https://orcid.org/0000-0001-7088-6191
\end{abstract}

\begin{abstract}
Resumo
Introdução. O envelhecimento é algo natural, caracterizado pela diminuição da funcionalidade do indivíduo. Objetivo. avaliar a relação da capacidade funcional e nível de depressão em idosos participantes de um grupo de convivência. Método. O estudo caracterizou-se por ser de natureza transversal com caráter descritivo e de abordagem quantitativa. Para coleta de dados utilizou-se um formulário sociodemográfico, e dois questionários, Índice de Katz e Escala de Depressão Geriátrica (GDS). Resultados. A amostra foi composta de 55 idosos, com a maior parte dos participantes sendo do sexo feminino $(n=$ 38). Ao observar a capacidade funcional constatou-se que o sexo feminino se mostrou mais dependente $(5,71 \pm 0,73)$ e apresentou maior sintomatologia depressiva comparada ao sexo masculino $(4,39 \pm 1,84)$. Com relação ao nível de depressão de maneira isolada pôde-se analisar que apenas $27,3 \%$ dos participantes apresentaram sintomatologia depressiva. Observou-se também uma correlação negativa e significante entre o Índice de Katz e GDS. Conclusão. Desta forma, conclui-se que a depressão afeta diretamente e de maneira negativa a vida de um idoso.
\end{abstract}

Unitermos. Envelhecimento; Depressão; Capacidade funcional

\footnotetext{
Abstract

Introduction. Aging is something natural, characterized by diminished functionality of the individual. Objective. To evaluate the relationship of functional capacity and level of depression in the elderly participants of a coexistence group. Method. The study was characterized by being of a cross-sectional nature with a descriptive character and a quantitative approach. A sociodemographic form and two questionnaires, Katz Index and Geriatric Depression Scale (GDS) were used for data collection. Results. The sample consisted of 55 elderly, with the majority of participants being female $(n=38)$. When observing the
} 
functional capacity, it was found that the female sex was more dependent $(5.71 \pm 0.73)$ and

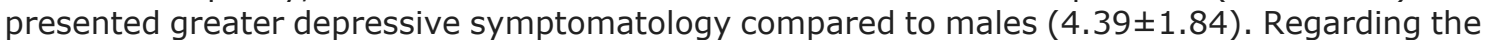
level of depression in isolation, it was possible to analyze that only $27.3 \%$ of the participants presented depressive symptomatology. There was also a significant negative correlation between the Katz Index and GDS. Conclusions. Thus, it is concluded that depression directly and negatively affects the life of an elderly person.

Keywords. Aging; Depression; Functional capacity

\section{Resumen}

Introducción. El envejecimiento es algo natural, caracterizado por una disminución de lafuncionalidad del individuo. Objetivo. Evaluar la relación entre capacidad funcional y nivel de depresión en personas mayores que participan em un grupo de convivência. Método. El estudio se caracterizó por ser de carácter transversal con carácter descriptivo y enfoque cuantitativo. Para la recolección de datos se utilizo um formulario sociodemográfico y dos cuestionarios, Índice de Katz y Escala de Depresión Geriátrica (GDS). Resultados. La muestra estuvo formada por 55 personas mayores, siendo la mayoría de los participantes mujeres $(n=38)$. Al observar la capacidad funcional, se encontró que las mujeres eran más dependientes $(5,71 \pm 0,73)$ y tenían mayores sintomas depressivos em comparación com los varones $(4,39 \pm 1,84)$. Encuanto al nivel de depresión de forma aislada, se pudo analizar que solo el $27,3 \%$ de los participantes presentaba sintomas depresivos. También hubo una correlación negativa y significante entre el índice de Katz y el GDS. Conclusiones. Así, se concluye que la depresión afecta directa y negativamente la vida de una persona mayor.

Palabras clave. Envejecimiento; Depresión; Capacidad funcional

Trabalho realizado na Faculdade Nova Esperança de Mossoró, FACENE RN, Mossoró-RN, Brasil.

Endereço para correspondência: Joelma G Silva. Faculdade Nova Esperança de Mossoró, FACENE RN. Av. Pres. Dutra, 701. Alto de São Manoel. CEP 59628-000. Mossoró-RN, Brasil. Fone: +55 84999300943. Email: fisiojoelmagomes@gmail.com

\section{INTRODUÇÃO}

De acordo com a Organização Mundial de Saúde (OMS), é considerado idoso nos países em desenvolvimento, aqueles a partir de 60 anos de idade; e nos países desenvolvidos, essa faixa etária gira em torno de 65 anos. Esse processo de envelhecer pode ser definido pela diminuição da funcionalidade do indivíduo e este comportamento está relacionado a mudança do perfil epidemiológico no país ${ }^{1-3}$. Neste cenário, se faz necessário uma discussão acerca da capacidade funcional desses indivíduos que está diretamente relacionada a execução das suas atividades 
físicas e mentais necessárias para preservação de suas atividades básicas de vida ${ }^{4}$.

Dessa forma, a perca desta capacidade está relacionada diretamente com a fragilidade e dependência e pode ser influenciada pela idade, presença de doenças crônicas, auto avaliação ruim de saúde, baixa renda e baixa escolaridade. Além de levar a uma maior susceptibilidade a hospitalização, sentimento de inutilidade e aumento do risco de morte ${ }^{5-7}$.

Diante deste estado de sofrimento surge uma tendência para o aparecimento da depressão, que pode ser definida como alterações psicopatológicas, sendo diagnosticada de acordo com a sintomatologia, gravidade, curso e prognóstico. Sua caracterização se dá pelo humor depressivo e/ou irritável, redução nas sensações de prazer ou alegria, variações do sono e apetite, desinteresse, entre outros. E É considerada a quarta causa de incapacitação social, sendo um problema de saúde pública ${ }^{8,9}$.

O Brasil, por exemplo, é o segundo país com mais registros de depressão da América, totalizando cerca de 11,5 milhões de indivíduos acometidos ${ }^{10}$. Sendo que na terceira idade este problema tem se tornado cada vez mais comum, afetando principalmente mulheres ou indivíduos que apresentem alguma morbidade, o que afeta diretamente 0 contexto social que ele está inserido ${ }^{11,12}$.

Diante do exposto, surge a necessidade de estudos que correlacionem essas variáveis na população idosa, para que a partir dos resultados possam surgir ações para amenizar os impactos causados por este quadro, bem como entender 
os fatores intervenientes nesses processos e traçar melhores planos tanto de prevenção como de reabilitação.

Portanto, o presente estudo teve como objetivo avaliar a relação da capacidade funcional e nível de depressão em idosos participantes de um grupo de convivência.

\section{MÉTODO}

\section{Amostra}

O presente estudo caracterizou-se por ser de natureza transversal com caráter descritivo e de abordagem quantitativa. Foi realizado em um grupo de convivência de idosos. A população do estudo foi composta por 100 idosos cadastrados no projeto, e a amostra foi obtida por conveniência, visto que a população dentro do grupo era bastante flutuante não estando em sua totalidade presente nas atividades proporcionadas. A coleta de dados foi realizada em três semanas totalizando um número de 55 indivíduos.

Foram incluídos na pesquisa indivíduos que fossem participantes do grupo, de ambos os sexos, com faixa etária a partir de 60 anos. Já com relação aos critérios de exclusão, foi levado em consideração indivíduos que apresentavam patologia incapacitante relacionada ao sistema musculoesquelético (a exemplo das artroses, artrites, degenerações musculares acentuadas e fundamentadas por laudos médicos, dentre outras) e/ou que já apresentassem algum quadro depressivo diagnosticado e atestado por um 
profissional competente. Além disso, foram excluídos aqueles indivíduos que não frequentavam de maneira regular o grupo ou estavam pela primeira vez na reunião no momento da coleta. Todos os indivíduos assinaram o Termo de consentimento livre esclarecido (TCLE) após explicação e leitura prévia.

Este projeto foi aprovado pelo comitê de ética e pesquisa da Universidade do Estado do Rio grande do Norte, sob o parecer: 3.035.896.

\section{Procedimento}

Após todo o procedimento de autorização, a coleta se realizou a partir de um formulário sociodemográfico, com 0 objetivo de coletar dados como: idade, sexo, escolaridade, raça, profissão, com quem convivem, patologias e uso de medicamento. Em seguida, aplicou-se a Escala de Depressão Geriátrica (GDS) elaborada por Yesavage e colaboradores no ano de 1983, com o intuito de avaliação do quadro depressivo em idosos, sendo composto de uma versão longa contendo 30 questões e uma versão curta que foi utilizada, composta de 15 questões que abordam desde a satisfação com a vida até a disposição destes indivíduos.

Para avaliar o nível de capacidade funcional dos indivíduos, foi utilizado o questionário Índice de Katz elaborado por Sidney Katz, no ano de 1963. Esse questionário avalia atividades consideradas básicas, tais como: banho, vestir-se, ir ao banheiro, transferência, continência e alimentação. A coleta de dados foi realizada 
em uma sala reservada, de forma individual. Devido algumas limitações da amostra, os questionários foram lidos pela pesquisadora e os participantes respondiam de forma oral, sendo marcada a alternativa desejada sem nenhuma interferência.

\section{Análise Estatística}

Os dados categóricos foram apresentados através de frequências simples e relativas. Logo após, a normalidade dos dados foi analisada através do teste de Shapiro Wilk com um nível de confiança de $(p<0,05)$. A partir disso, os dados foram apresentados por meio de medidas de tendência central e de dispersão (média e desvio padrão). Em seguida, aplicou-se o teste $\mathrm{T}$ para amostras independentes e o teste de correlação $\mathrm{R}$ de Pearson. Para todas as análises, considerou-se um nível de significância de $p<0,05$.

\section{RESULTADOS}

Os resultados a seguir, referem-se a uma amostra de 55 indivíduos idosos, que são participantes de um grupo de convivência. Diante disto, observa-se na Tabela 1 os dados sociodemográficos com a maior parte dos idosos possuindo o ensino fundamental incompleto $(80 \%)$, sendo de raça parda $(69,1 \%)$, com profissão de agricultor $(52,7 \%)$, convivendo com seus familiares $(78,1 \%)$. Com relação aspectos clínicos, um maior número não apresentou nenhuma patologia associada ao sistema 
musculoesquelético $(49,1 \%)$, porém fazem uso de medicamento para o tratamento de doenças crônicas $(85,5 \%)$.

Tabela 1. Caracterização da amostra.

\begin{tabular}{|c|c|c|}
\hline & $\begin{array}{c}\text { Masculino }(n=17) \\
n(\%)\end{array}$ & $\begin{array}{c}\text { Feminino }(n=38) \\
n(\%)\end{array}$ \\
\hline \multicolumn{3}{|l|}{ Escolaridade } \\
\hline Analfabeto & $3(5,5)$ & $1(1,8)$ \\
\hline $\begin{array}{l}\text { Ensino Fundamental } \\
\text { Incompleto }\end{array}$ & $13(23,6)$ & $31(56,4)$ \\
\hline $\begin{array}{l}\text { Ensino Fundamental } \\
\text { Completo }\end{array}$ & $0(0,0)$ & $1(1,8)$ \\
\hline Ensino Médio Completo & $1(1,8)$ & $5(9,1)$ \\
\hline \multicolumn{3}{|l|}{ Raça } \\
\hline Negra & $2(3,6)$ & $5(9,1)$ \\
\hline Branca & $3(5,5)$ & $7(12,7)$ \\
\hline Parda & $12(21,8)$ & $26(47,3)$ \\
\hline \multicolumn{3}{|l|}{ Profissão } \\
\hline Agricultor (a) & $13(23,6)$ & $16(29,1)$ \\
\hline Dona do lar & $0(0,0)$ & $11(20,0)$ \\
\hline Artesão & $0(0,0)$ & $6(10,9)$ \\
\hline Professor (a) & $0(0,0)$ & $3(5,5)$ \\
\hline Outros & $4(7,3)$ & $2(3,6)$ \\
\hline \multicolumn{3}{|l|}{ Convive com } \\
\hline Familiares & $13(23,6)$ & $30(54,5)$ \\
\hline Sozinho & $4(7,3)$ & $8(14,5)$ \\
\hline Outros & $0(0,0)$ & $0(0,0)$ \\
\hline \multicolumn{3}{|l|}{ Patologias Associadas } \\
\hline Não possui & $11(20,0)$ & $16(29,1)$ \\
\hline Artrose & $1(1,8)$ & $9(16,4)$ \\
\hline Dores não diagnosticadas & $3(5,5)$ & $8(14,5)$ \\
\hline Outras & $2(3,6)$ & $5(9,1)$ \\
\hline \multicolumn{3}{|l|}{ Faz Uso de } \\
\hline \multicolumn{3}{|l|}{ Medicamentos } \\
\hline $\operatorname{Sim}$ & $14(25,5)$ & $33(60,0)$ \\
\hline Não & $3(5,5)$ & $5(9,1)$ \\
\hline
\end{tabular}


Pôde-se observar que o perfil dos indivíduos participantes deste grupo de convivência são idosos ativos, que ainda realizam alguma atividade laboral, e afirmam participar do grupo para distração e ocupação de seus horários vagos. Esses idosos relataram ainda gostar das atividades propostas pelo coordenador e se sentem muito acolhidos neste ambiente.

Nesse contexto, quando avaliado a questão da capacidade funcional e da depressão a partir dos valores médios, conforme evidenciado na Tabela 2, não foram detectadas diferenças significantes de acordo com o sexo.

Tabela 2. Comparação dos valores do índice Katz e o escore total do GDS.

\begin{tabular}{cccc}
\hline & $\begin{array}{c}\text { Masculino }(\mathbf{n = 4 3}) \\
\text { Média } \pm \text { DP }\end{array}$ & $\begin{array}{c}\text { Feminino }(\mathbf{n = 1 2}) \\
\text { Média } \pm \text { DP }\end{array}$ & Valor de $\mathbf{p}$ \\
\hline Idade & $74,18 \pm 8,94$ & $70,42 \pm 6,66$ & 0,089 \\
Índice Katz & $6,00 \pm 0,01$ & $5,71 \pm 0,73$ & 0,679 \\
Escore GDS & $4,18 \pm 1,70$ & $4,39 \pm 1,84$ & 0,111 \\
\hline
\end{tabular}

Porém, é possível observar que o sexo masculino apresentou uma média discretamente maior com relação à capacidade funcional $(6,00 \pm 0,01)$, ou seja, mais independentes quando comparados ao sexo feminino. Relativamente, o sexo feminino mostrou uma maior média no Escore GDS comparado ao sexo masculino $(4,39 \pm 1,84)$, ou seja, uma tendência maior para depressão. 
Quando se analisa a suspeita de depressão, a partir da aplicação do GDS, foi possível observar, como mostra a Figura 1, que aqueles indivíduos com suspeita de depressão foi minoria $(27,3 \%)$. Este fato pode ser explicado pelo contexto que esses sujeitos estão inseridos, fazendo parte de um grupo de convivência o que possivelmente estar interferindo positivamente em suas vidas.

Figura 1. Número de idosos com sintomas relacionados a depressão.

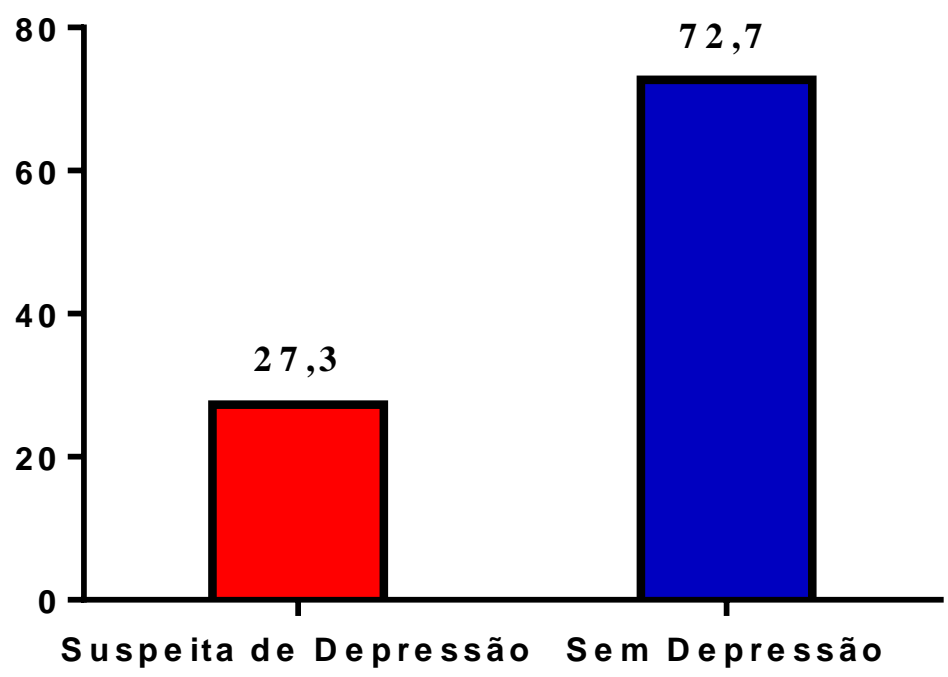

Já com relação aos valores apresentados na tabela 3, que apontam para uma correlação negativa e significante entre o escore total do GDS e índice Katz ( $r=-0,324$; $p=0,016)$, indicando que o número de sintomas depressivos afeta negativamente a capacidade funcional. Sobre isso, é 
válido destacar que dos 55 idosos, 21 (27,3\%) apresentaram sintomas relacionados à depressão.

Tabela 3. Correlação entre o índice Katz e o escore total do GDS.

\begin{tabular}{ccc} 
& Escore total GDS \\
& $\mathrm{r}$ & Valor de $\mathrm{p}$ \\
\hline Idade & 0,091 & 0,507 \\
Índice Katz & $-0,324^{*}$ & $0,016^{*}$ \\
\hline * valor de $\mathrm{p}<0,05$ &
\end{tabular}

Esses dados levantam uma preocupação acerca da situação desses idosos, à medida que há uma influência em todo o contexto que eles estão inseridos, impactando negativamente e tornando-o mais dependente em suas Atividades Básicas de Vida Diária (ABVD's).

\section{DISCUSSÃO}

O idoso sente motivação para participar de grupos de convivência, pois grande parte não pratica atividades sociais e não possuem atividades relacionadas ao lazer, passando assim a maior parte do tempo em casa ${ }^{13}$. Nesse contexto, há um predomínio característico dos perfis do sexo feminino, sendo justificado pelo fato de as mulheres ainda apresentarem maior atenção com a saúde e com 0 autocuidado do que o sexo masculino ${ }^{6}$ concordando assim com o presente estudo, onde a predominância dos participantes foi do sexo feminino. 
Com relação ao sintoma depressão, alguns autores relataram em estudo realizado em centros de convivência para idosos ${ }^{15}$, que apenas uma pequena parte dos participantes apresentaram sintomas depressivos, o que desponta para um papel social importante desses lugares na vida do idoso.

Isto se torna relevante, pois o envelhecimento já está associado ao surgimento de doenças crônicas, sendo um ciclo do processo envelhecer, e dentre estas doenças a depressão é a principal delas ${ }^{12}$. Porém, no presente estudo, a maioria dos participantes não apresentaram sintomatologia depressiva de acordo com suas respostas, reafirmando o importante papel social do grupo na vida desses indivíduos.

Essa discussão se faz necessária, pois todo o quadro sintomatológico depressivo pode impactar negativamente na qualidade e contexto da vida desses idosos, o que afeta diretamente sua capacidade de lidar com o mundo, como também a sua funcionalidade ${ }^{8}$. Isto concorda com a presente pesquisa, pois foi possível encontrar uma correlação negativa entre essas variáveis.

Ainda concordando com este estudo, a literatura ${ }^{15}$ aponta que em idosos institucionalizados, a capacidade funcional foi consistentemente preditora do escore de depressão. Porém, em outra pesquisa, quando se trata de capacidade funcional de maneira isolada, não houve no contexto diferença estatisticamente significativa relacionada ao sexo e a alguma ABVD. Porém o sexo feminino mostrou 
uma maior dependência na realização de algumas atividades; padrão apontado pela literatura ${ }^{6}$. Este fato pode estar associado a questão dos idosos homens se mostrarem mais ativos, como foi encontrado neste estudo com relatos de ter mais disposição para o trabalho, diferente do sexo feminino que apontaram preferir ficar em casa, cuidando de seus afazeres domésticos, e assistindo TV, ao invés de sair e se relacionar com o mundo exterior.

Diante desta discussão, torna-se evidente que os grupos de convivência influenciam positivamente na vida dos indivíduos idosos, estando relacionados a menor porcentagem da sintomatologia depressiva e consequentemente contribuindo também para uma melhor capacidade funcional.

\section{CONCLUSÃO}

A partir do presente estudo, foi possível perceber uma correlação negativa entre o nível de depressão e a capacidade funcional em idosos sendo possível inferir que a depressão afeta diretamente e de maneira negativa a vida de um idoso.

A presença da sintomatologia depressiva foi identificada com valores discretos, estando mais presente no sexo feminino. Atrelado a isto, foi possível inferir por meio da avaliação da capacidade funcional, que o sexo masculino se apresentou mais independente quando comparado ao sexo feminino. 
Desta forma, destaca-se a necessidade de incentivar a participação desses indivíduos a esse tipo de programa no sentido de oferecer um melhor contexto de vivência para essa população.

\section{REFERÊNCIAS}

1.Inagaki RK, Yamaguchi MH, Kassada D, Matsuda LM, Marcon SS. A vivência de uma idosa cuidadora de um idoso doente crônico. Cienc Cuidado Saúde 2013;7:1-4.

http://dx.doi.org/10.4025/cienccuidsaude.v7i0.20802

2.Lima AP, Delgado EI. A melhor idade do Brasil: aspectos biopsicossociais decorrentes do processo de envelhecimento. Ulbra Mov - Rev Edu Fis 2010;1:76-91.

http://www.periodicos.ulbra.br/index.php/actabrasileira/article/view/ 3063.

3.Veras RP, Oliveira M. Envelhecer no Brasil: a construção de um modelo de cuidado. Cienc Saude Col 2018;23:1929-36. http://dx.doi.org/10.1590/1413-81232018236.04722018

4.Gama MFL, Vieira JNL, Carvalho STRF. Efeitos de um programa cinesioterapêutico na autonomia funcional de idosas. Revista CEUMA perspectivas 2016;27:7-17. https://doi.org/10.24863/rccp.v27i1.37 5. Nunes JD, Saes MO, Nunes BP, Siqueira FC, Soares DC, Fassa ME, et al. Indicadores de incapacidade funcional e fatores associados em idosos: estudo de base populacional em Bagé, Rio Grande do Sul. Epidemiol Ser Saúde 2017;26:295-304. http://dx.doi.org/10.5123/s1679-49742017000200007.

6.Araújo GK, Sousa RC, Souto RQ, Júnior EG, Eulálio MC, Alves FA, et al. Capacidade funcional e depressão em idosos. Rev Enferm 2017;11:3778-86. http://dx.doi.org/10.5205/reuol.12834-30982-1SM.1110201711

7.Dodig S, Cepelak I, Pavick I. Caracteristicas da senescência e envelhecimento. Biochem

Med 2019;29:1.

http://dx.doi.org/10.11613/BM.2019.030501

8. Lima AM, Ramos JL, Bezerra IM, Rocha RP, Batista HM, Pinheiro WR. Depressão em idosos: uma revisão sistemática da literatura. Rev Epidemiol Controle Infec 2016;2:96-103. http://dx.doi.org/10.17058/reci.v6i2.27.

9.Magalhães J, Carvalho A, Carvalho S, Alencar D, Moreira W, Parente A. Depressão em idosos na estratégia saúde da família: uma contribuição para a atenção primária. Rev Min Enferm 2016;20:16. http://dx.doi.org/10.5935/1415-2762.20160016

10. Mendes GL, et al. Terapêuticas medicamentosas e exercícios físicos na prevenção e tratamento de depressão em idosos: revisão 
sistemática. Arq Bras Ed Fis 2020;3:43-56.

http://dx.doi.org/10.20873/abef.2595-0096.v3n1p43.2020

11.Nery BL, Cruz KC, Faustino AM, Santos CT. Vulnerabilidades, depressão e religiosidade em idosos internados em uma unidade de emergência. Rev

Gaúcha

Enferm

2018;39:1-10.

http://dx.doi.org/10.1590/1983-1447.2018.2017-0184.

12. Lima CM, Barros NS, Barroso BM, Pereira AC, Silva AP. Um estudo sobre depressão na terceira idade. Inova Saúde 2018;7(1):27-39. http://dx.doi.org/10.18616/is.v7i1.4258

13.Xavier LN, Sombra IC, Gomes AM, Oliveira GL, Aguiar CP, Sena RM. Grupo de convivência de idosos: apoio psicossocial na promoção da saúde. Rev Rede Enferm NE 2015;16:557-66. http://dx.doi.org/10.15253/2175-6783.2015000400013

14. Oliveira DA, Gomes L, Oliveira RF. Prevalência de depressão em idosos que frequentam centros de convivência. Rev Saúde Publ 2006;40:734-6.

http://dx.doi.org/10.1590/S0034-

$\underline{89102006000500026}$

15.Silva JK, Albuquerque AC, Souza EM, Monteiro FS, Esteves GL. Sintomas depressivos e capacidade funcional em idosos institucionalizados. Rev Cult Cuidados 2015;19:157-67. http://dx.doi.org/10.14198/cuid.2015.41.18 